\title{
Snow avalanche speed determination using seismic methods
}

\author{
I. Vilajosana ${ }^{\text {a,* }}$, G. Khazaradze ${ }^{\text {a }}$, E. Suriñach ${ }^{a}$, E. Lied ${ }^{b}$, K. Kristensen ${ }^{b}$ \\ ${ }^{a}$ Grup d'Allaus (RISKNAT); Dept. de Geodinàmica i Geofisica, Universitat de Barcelona, Martí i Franquès s/n, 08028, Barcelona, Spain \\ ${ }^{\mathrm{b}}$ Norwegian Geotechnical Institute, Postbox 3930 Ullevaal Stadion, N-0806, Oslo, Norway
}

\section{Abstract}

We present a new method to determine the average propagation speed of avalanches using seismic techniques. Avalanche propagation speeds can reach $70 \mathrm{~m} / \mathrm{s}$ and more, depending on a wide range of factors, such as the characteristics of the avalanche track (e.g. topography) and the snowpack properties (e.g. density). Since the damage produced by the avalanche depends primarily on the size and on the speed of the avalanche, the knowledge of the latter is therefore crucial for estimating avalanche induced hazard in inhabited mountain areas. However, our knowledge of this basic physical parameter is limited by the difficulty of conducting various measurements in the harsh winter weather conditions that often accompany this natural phenomenon.

The method of avalanche speed determination presented in this paper is based on cross-correlation and time-frequency analysis techniques. The data used in this study come from the Ryggfonn (Norway) avalanche experimental site operated by the Norwegian Geotechnical Institute (NGI), and recorded by an array of 6 geophones buried along the main avalanche path during the 2003-2004 and 2004-2005 winter seasons. Specifically, we examine the speeds of 11 different events, characterized by size and snow type. The results obtained are compared with independent speed estimates from CW-radar and pressure plate measurements. As a result of these comparisons our method was validated and has proved to be successful and robust in all cases. We detected a systematic behaviour in the speed evolution among different types of avalanches. Specifically, we found that whereas dry/mixed type flow events display a complex type of speed evolution in the study area with a gradual acceleration and an abrupt deceleration, the speed of the wet snow avalanches decreases with distance in an approximately linear fashion. This generalization holds for different size events.

In terms of time duration and maximum speed of the studied events, dry/mixed type avalanches lasted between 8 to $18 \mathrm{~s}$ and reached speeds up to $50 \mathrm{~m} / \mathrm{s}$, whereas the duration of wet avalanches ranged between 50 and $80 \mathrm{~s}$ and their maximum speeds were $10 \mathrm{~m} / \mathrm{s}$.

(C) 2006 Published by Elsevier B.V.

Keywords: Seismic record; Snow avalanche; Time-frequency analysis; Speed

\section{Introduction}

Land use planning based on hazard mapping is the most cost-effective way to mitigate avalanche risk in

\footnotetext{
* Corresponding author. Fax: +34 934021340 .

E-mail address: vilajosana@ub.edu (I. Vilajosana).
}

inhabited areas. This procedure usually involves estimating the run-out distance of hypothetical avalanches with long return periods and is usually achieved with numerical models based on fluid dynamics equations (Harbitz et al., 1998). One of the main output parameters of these models is the avalanche speed. The speeds obtained from the data analysis of large scale 
experiments are compared with those obtained by the models in order to calibrate them.

Avalanche speeds depend on a wide range of factors, including the characteristics of the track (e.g. topography) and of snowpack properties (e.g. grain/clod size and density). Avalanches can reach speeds up to $70 \mathrm{~m} / \mathrm{s}$ and more. Since the damage produced by the avalanche depends on its propagation speed, the knowledge of this parameter is crucial for estimating avalanche induced hazards in inhabited mountain areas. However, our knowledge of this basic physical parameter is limited by the difficulty of conducting measurements, which is compounded by the harsh weather conditions that often accompany snow avalanches and by the complexity of the physical phenomenon itself.

Earlier studies of speed determination of snow avalanches have been based on the processing of video images (Granada et al., 1995; McElwaine, 2004), the determination of internal clod speeds using LED-photocells (Dent et al., 1998), on the interpretation of data from pressure load cells (Norem et al., 1985), and Doppler-radar (Gubler, 1987) techniques among others. The few studies of avalanche speed determination based on seismic methods include works by Schaerer and Salway (1980), Nishimura et al. (1993), and Nishimura and Izumi (1997), who used basic picking techniques to obtain the arrival time of the avalanche body over geophones installed along the avalanche path. However, none of these studies describe in detail the criteria of the selection techniques used.

In this paper we present a new method for determining the average propagation speed of snow avalanches from seismic time series. Specifically, we present the averaged speed values of the avalanche front obtained for different avalanches, and their evolution with distance along the main propagation path. The data are from the Ryggfonn avalanche experimental site in Norway operated by the Norwegian Geotechnical Institute (NGI) (Lied et al., 2002). They were obtained from a set of 6 geophones and from independent sources such as continuous wave Doppler-radar (CW-Doppler radar) and pressure Load Plates (LP) measurements (Gauer et al., 2004).

The methods for determining the avalanche speed presented in this paper are based on cross-correlation and time-frequency analyses of the data recorded by seismic sensors.

For the last decade our group at the Universitat de Barcelona (UB) has been studying the seismic signals generated by avalanches at different sites in Europe. The most important results obtained in these studies are related to reproducibility, time evolution of the seismic signal spectra, and identification of various sources of seismic energy (e.g. Biescas et al., 2003; Suriñach et al., 2000). The method of speed determination of avalanches presented in this paper is a continuation of these previous studies.

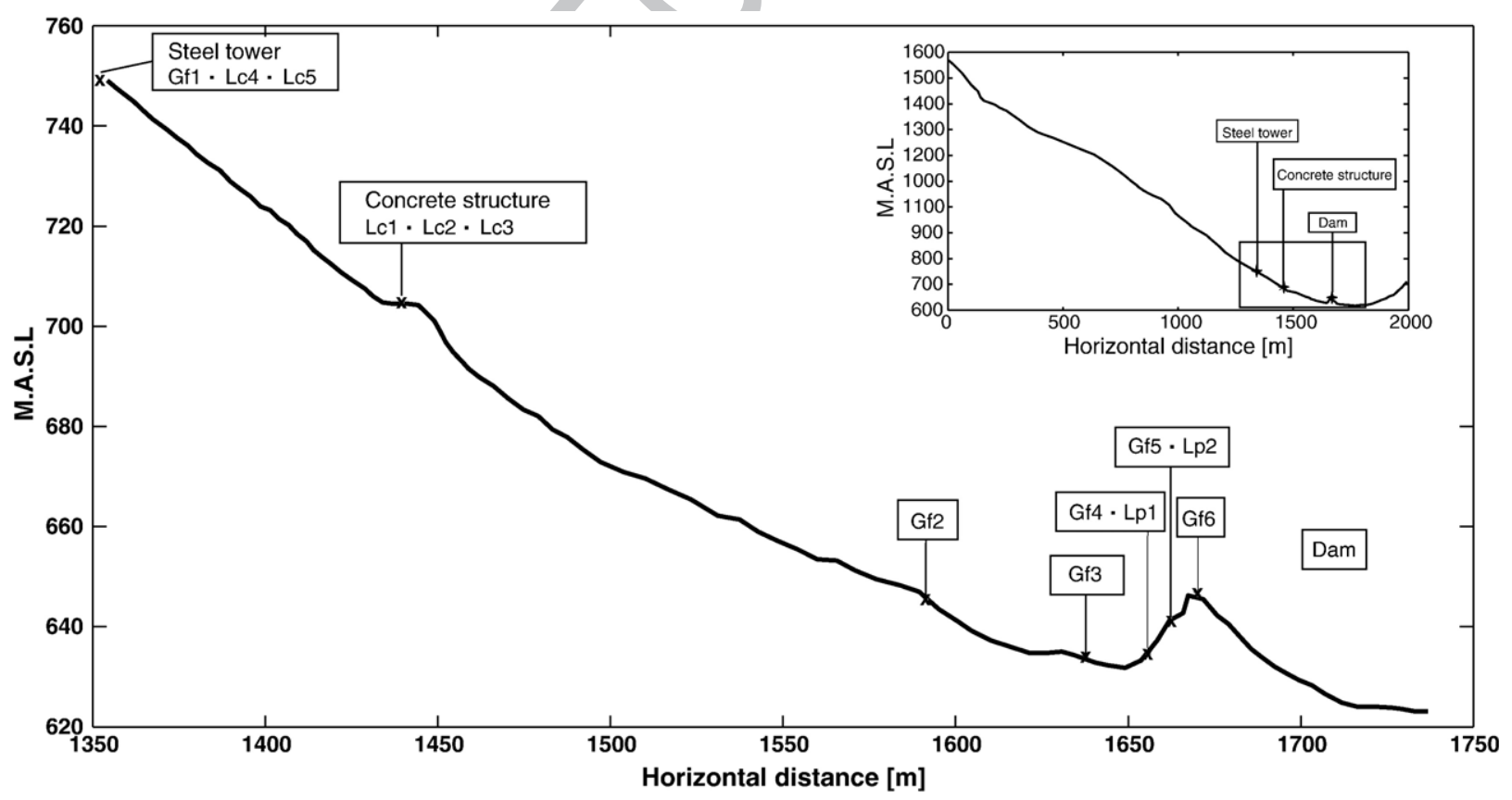

Fig. 1. Detailed profile of the lowest part of the avalanche track in Ryggfonn including the installed instrumentation. The inset in the upper right corner shows the complete Ryggfonn avalanche path profile. 


\section{Experimental site and data}

The Ryggfonn full-scale avalanche site, situated $500 \mathrm{~km}$ north-west of Oslo, Norway, was set up to study snow avalanches by the NGI in 1980 (Lied et al., 2002). Since then, approx. 2-3 avalanches per year have been released at the site. The avalanche path at Ryggfonn has a drop of $900 \mathrm{~m}$ and a horizontal length of $2100 \mathrm{~m}$ (Fig. 1). The avalanches released at the site range in volume between $10,000 \mathrm{~m}^{3}$ and $500,000 \mathrm{~m}^{3}$, reaching maximum velocities of $60 \mathrm{~m} / \mathrm{s}$. In the lower part of the avalanche path there is a retaining dam of unconsolidated material, $16 \mathrm{~m}$ in height and $75 \mathrm{~m}$ in crown length. This dam is equipped with different instruments (Fig. 1). Two 3D-load plates (LP1, LP2) are placed at the front. Three 1D-4.5 Hz vertical component geophones (GF4, GF5, and GF6) are embedded in the dam. Two of these (GF4 and GF5) are placed beside each load plate. A $6.5 \mathrm{~m}$ steel mast with a horizontal uni-axial geophone (HG1) located on top of the dam completes the installation. In the avalanche path, two more geophones, spaced $47.4 \mathrm{~m}$ apart, are buried in the ground (GF2, GF3) (Fig. 1). A $4.5 \mathrm{~m}$ high concrete structure containing three load cells (LC1, LC2, and LC3) is placed approx. $230 \mathrm{~m}$ up-slope from the dam in the main avalanche path. A $5.5 \mathrm{~m}$ high cylindrical steel tower equipped with two more load cells (LC4, LC5) and one geophone (GF1) with the same characteristics as the others is placed $90 \mathrm{~m}$ above this structure.

A shelter containing the control and recording instruments is located in the valley $500 \mathrm{~m}$ east of the dam, and is provided with power, telephone line and ISDN connection. The recording system is triggered by geophone GF1 when the avalanche front hits the steel tower. All the measurements from the different instruments are recorded at a sampling rate of $150 \mathrm{sps}$ with a local common base of time. The total length of the records is $150 \mathrm{~s}$ including $25 \mathrm{~s}$ of pre-triggered data.

During the 2003-2004 winter season a frequency modulated continuous wave Doppler radar (CW-Doppler radar) was also operational (Sigurðsson et al., 2004).

In this study we analysed the data from 11 avalanches that occurred during the winter seasons 2003-2004 and 2004-2005 and one from 2000 (Table 1). The avalanches differed in size and type of snow. All the avalanches with the exception of events 6 and 7 were triggered naturally. Unfortunately, the availability of complementary information including field observations and video images is limited. Seismic and load plate data analyses indicated that in avalanche 2 the dense body did not reach the dam although the aerosol passed over the dam. Avalanche 4 did not reach the dam (field
Table 1

List of studied events

\begin{tabular}{|c|c|c|c|c|c|}
\hline $\begin{array}{l}\text { No. } \\
\text { event }\end{array}$ & Date & $\begin{array}{l}\text { hh:mm } \\
\text { (local time) }\end{array}$ & Release & Type $^{a}$ & Size $^{\mathrm{b}}$ \\
\hline 1 & $2003 / 12 / 15$ & $16: 35$ & Natural & Dry/mixed & Medium (3) \\
\hline 2 & $2003 / 12 / 17$ & $03: 14$ & Natural & Dry/mixed & Medium (3) \\
\hline $3 a$ & $2004 / 02 / 04$ & $06: 10$ & Natural & Wet & Medium (3.5) \\
\hline $3 b$ & $2004 / 02 / 04$ & $06: 12$ & Natural & Wet & Medium (3.5) \\
\hline 4 & $2004 / 02 / 24$ & $08: 50$ & Natural & Dry/mixed & Small (2) \\
\hline $5 a$ & $2004 / 02 / 24$ & $22: 30$ & Natural & Dry/mixed & Medium (3) \\
\hline $5 b$ & $2004 / 02 / 24$ & $22: 31$ & Natural & Dry/mixed & Medium (3) \\
\hline 6 & $2004 / 02 / 28$ & $15: 22$ & Artificial & Dry/mixed & Medium (3.5) \\
\hline 7 & $2000 / 02 / 17$ & $13: 55$ & Artificial & Dry/mixed & Large (4) \\
\hline $8 \mathrm{a}$ & $2005 / 01 / 07$ & $04: 17$ & Natural & Dry/mixed & Medium (3.5) \\
\hline $8 b$ & $2005 / 01 / 07$ & $04: 18$ & Natural & Wet & Medium (3.5) \\
\hline
\end{tabular}

observation). The two natural events at 02/04/2004 triggered the data acquisition system at an interval of 160 s. Given that the generated seismic signal of these events is continuous without interruption for $230 \mathrm{~s}$, it is difficult to ascertain whether these correspond to two independent avalanches or to one avalanche with two parts. In our study we regard them as independent wet flow avalanches, denoted as events $3 \mathrm{a}$ and $3 \mathrm{~b}$ to ease the subsequent processing. During the night of the 02/24/ 2004 two triggers ( $5 a$ and $5 b$ ) occurred within an interval of $40 \mathrm{~s}$. We treated them as independent avalanches because the events were clearly separated by a period of very low signals in the time series. In the afternoon of 01/07/2005 two consecutive triggers recorded a naturally released avalanche. After studying the signals it is reasonable to assume that the avalanche consists of two different parts (dry/mixed part and wet/ dense flow).

\section{Data analysis}

The main principle of the presented method of avalanche speed determination is based on the difference in the arrival times of the avalanche over two separate seismic sensors. To this end, we applied the procedure described below based on the characteristics of the seismic wave propagation. In our approach, it is assumed that at least the front of the avalanche reaches the geophone.

The running spectrum (RS) of the signal is studied prior to determining the avalanche arrival time over a sensor in the time series. In the RS, a selection of the time interval corresponding to the first window showing the highest amplitudes in the high frequency content of 
180 the spectra is performed as presented in Fig. 2b. We 181 attribute this time interval to the arrival of the avalanche 182 front over the sensor. The increase in the amplitude in 183 the RS with time is produced by the reduction of the 184 distance source-receiver as the avalanche front 185 approaches the sensor (Suriñach et al., 2005). This is 186 physically supported by the anelastic attenuation of 187 seismic waves with distance (Aki and Richards, 1980), 188 and is corroborated by the results of earlier studies. In 189 these studies we demonstrated that the peaks of 190 maximum seismic energy in the high frequency content 191 of the time series are related to snow erosion over the geophone. These results were obtained from the correlation of data from FMCW radar and from seismic sensors placed together in the avalanche path at the Vallée de la Sionne experimental site in Switzerland (Biescas, 2003; Biescas et al., 2002).

The selection of the window is performed by means of 1) computation of the running spectrum (RS) of the seismic time series based on short time Fast Fourier Transform (FFT) (Brigham, 1974). We used a Hanning window and an FFT length of 128 samples $(0.85 \mathrm{~s})$ with a $50 \%$ overlapping window taking into account the data sampling rate $(150 \mathrm{sps})$ and the trade-off between time
192
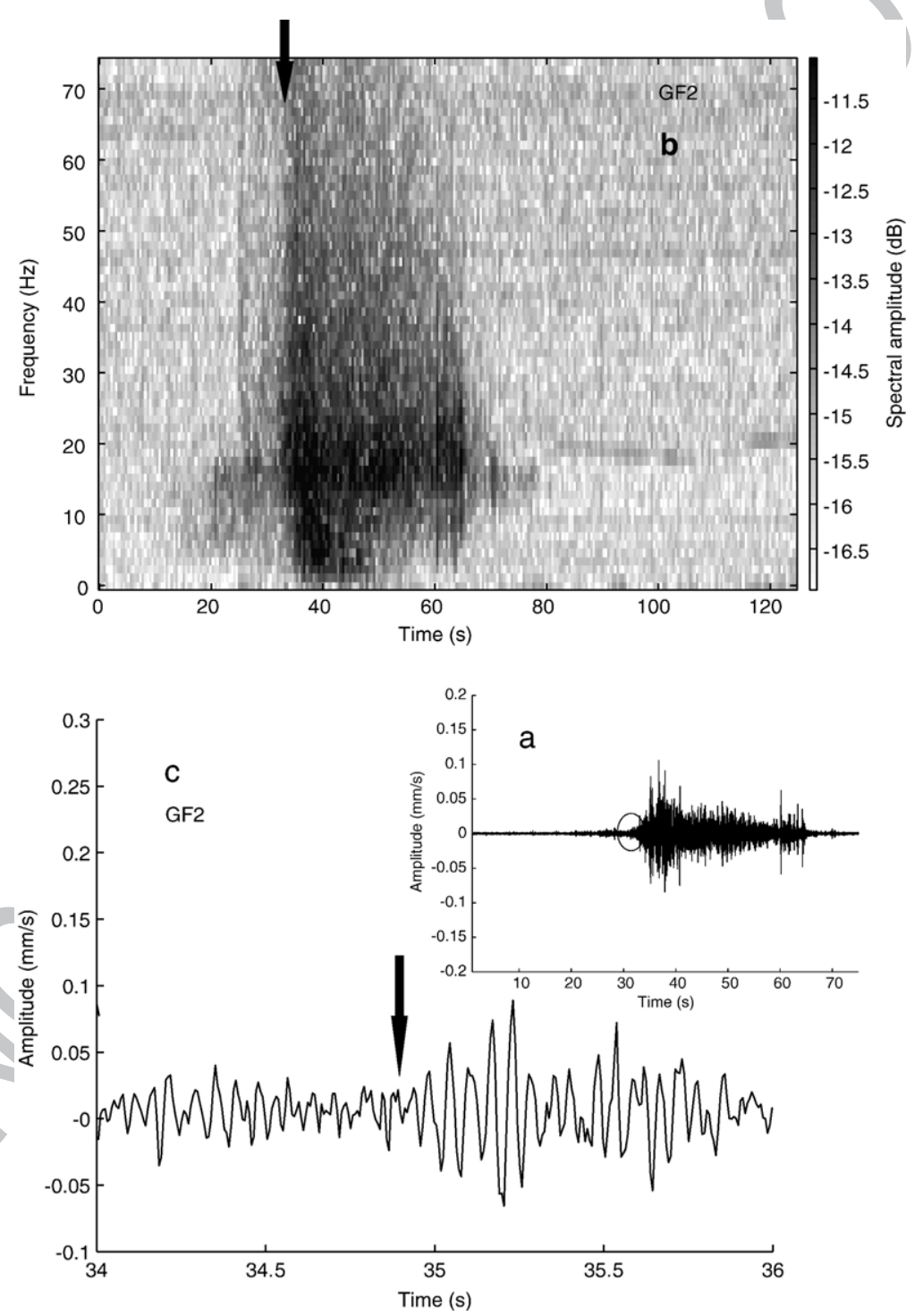

Fig. 2. a) GF2 time series of the avalanche released on 2004/02/28. b) Running spectra, the arrow indicates the selected time window corresponding to the arrival of the avalanche front over GF2. c) Detail of the time series window pre-selected in the RS, the arrow indicates the time of the avalanche arrival over GF2. 
204 and frequency resolutions (Flandrin, 2002); and 2) 205 selection of the first time window showing maximum 206 amplitudes at the highest frequencies in the RS (Fig. 2b). 207 The arrival time in the seismic time series is 208 performed by picking a discontinuity in the amplitude 209 or/and frequency of the time series in the selected 210 window using the standard seismological technique 211 (PK) (Fig. 2c). However, there are no characteristic 212 features to identify the arrival of the front.

As a result of this procedure the arrival time of the avalanche front at each geophone is obtained. Subsequently, using the distance between the geophones the average propagation speed of the avalanche between the pairs of geophones is obtained. When the PK procedure is not easy due to the lack of clarity of the wave arrival as in the case of small avalanches which generate weak vibrations or in the case of signals produced by slow dense avalanches recorded in the sensors located in the dam (GF4 and GF5) (further discussion is presented below) an alternative, cross-correlation procedure (XC) is applied.

The XC procedure consists of determining the lag time between the previously selected seismic time series windows corresponding to two separate geophones. The lag time, which indicates the time shift between the two time series, yields the difference in arrival time of the avalanche front needed to obtain the average propagation speed of the avalanche between the two points (Fig. 3). The XC method assumes that signals generated by the same source must be comparable. Earlier studies demonstrate that the main sources of the seismic signals are snow erosion, changes in the slope and impacts with obstacles (Suriñach et al., 2001). In our case, the signals to be identified are mostly produced by snow erosion, which is assumed to occur mainly near the front of the avalanche (Gauer and Issler, 2004).

The cross-correlation was performed on a windowed subset of the seismic time series. The time windows to be correlated are selected considering 5-10 s prior to the selected time in the RS. This window includes the length of the total time scale of the vortex inside the avalanche front (McElwaine, in press) and the signal before the arrival of the avalanche front over the sensor. Correlation of a longer time series provides no information on the propagation of the avalanche front. Rather, it reflects the specifics of the propagation path and the whole event. The selection of the window demands a prior detailed analysis of the signal, taking into account the shape of each time series to identify the part of the signal produced by the approaching avalanche and not by distant impacts, which are easily identifiable because they are observed almost simultaneously in all the time series. Correlations with
230 231
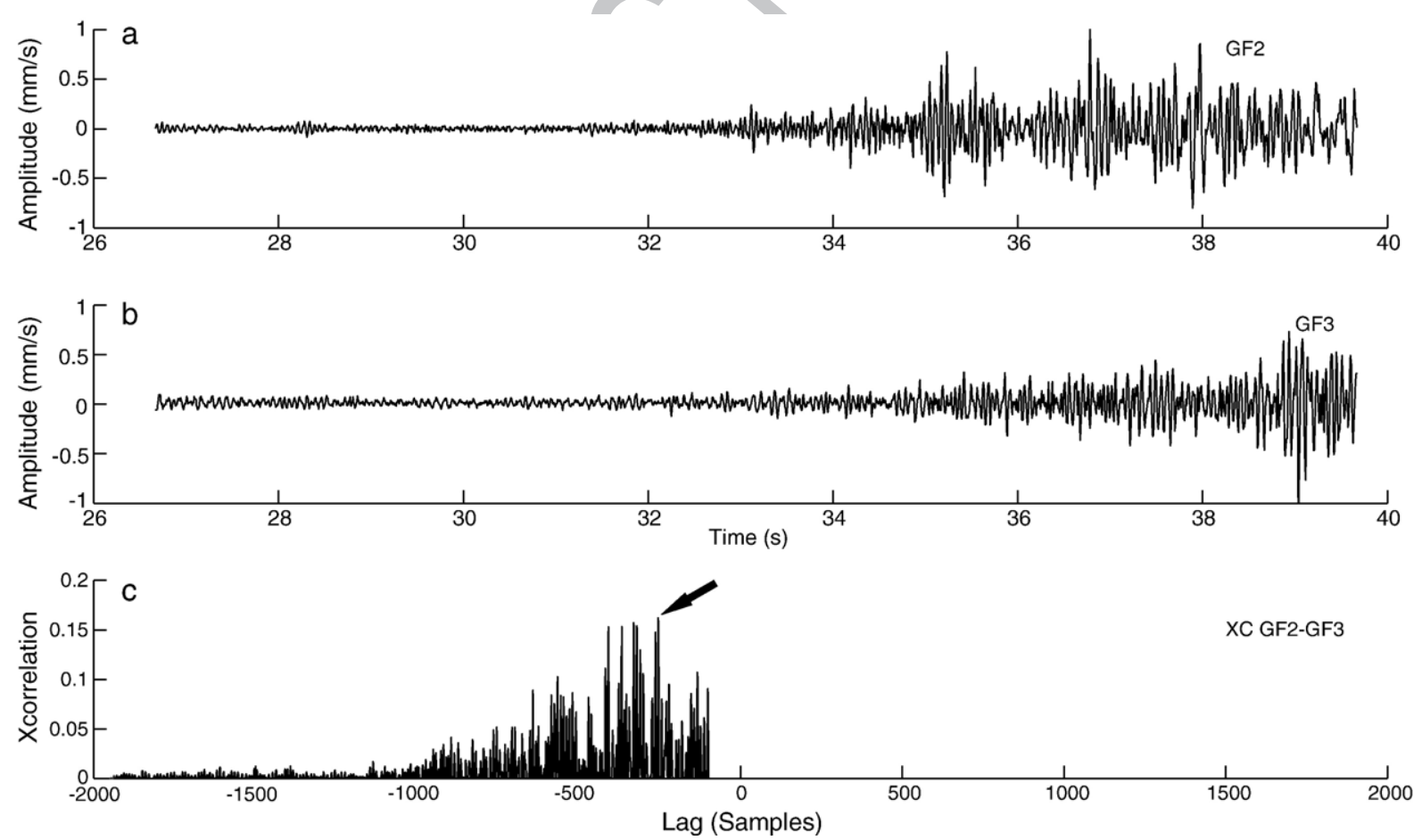

Fig. 3. a) and b) Time series windows of the avalanche released on 2004/02/28 for cross-correlation. c) Cross-correlation of the time series. The arrow indicates the selected lag time. 
256 positive lag times are excluded since these would imply

257 an upward propagation of the avalanche. Also excluded

258 are the lag times that give unrealistic speeds $(<1$ and

$259>70 \mathrm{~m} / \mathrm{s}$, for the Ryggfonn path), which can be attributed
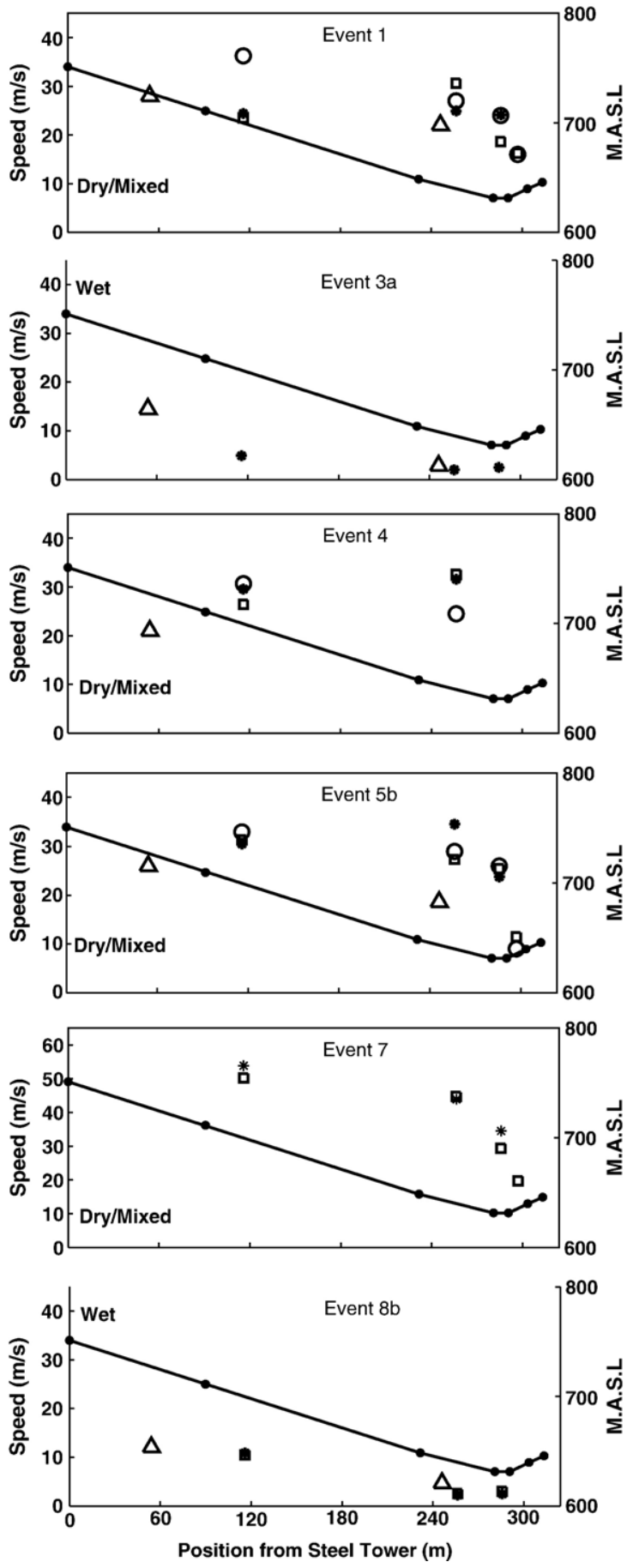

to the propagation of sound and/or ground vibrations rather than the snow avalanche.

The XC method involves pairs of seismic time series. As a consequence, we were able to obtain the differences
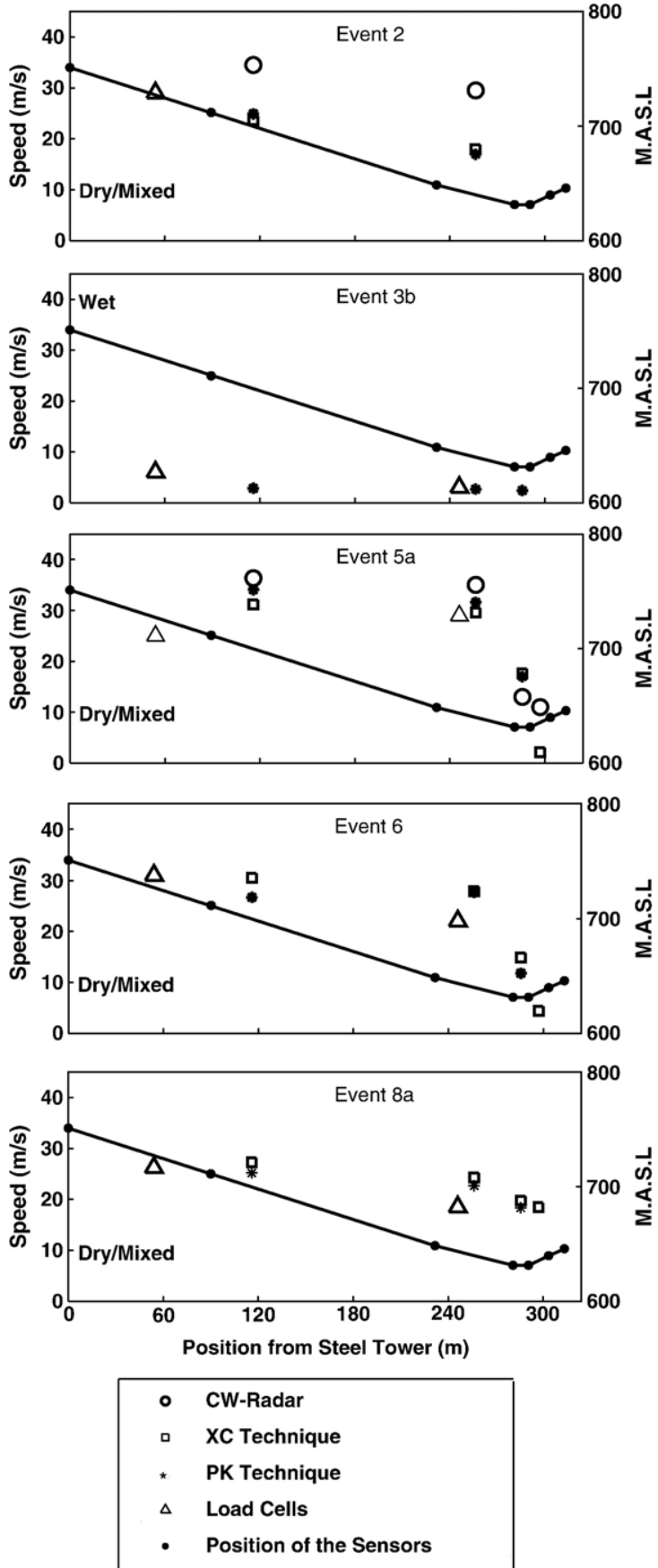

Fig. 4. Comparison of the speeds of 11 avalanches released at Ryggfonn obtained from the data of various instruments (CW-radar, geophones, load plates). Continuous lines shows the path profile and dots indicate the position of the measuring instruments. The speeds are presented at the midpoint between adjacent instrument locations. 
264 between the arrival times for four pairs of sensors (GF1265 GF2, GF2-GF3, GF3-GF4 and GF4-GF5). This is not the 266 case with the PK method with which we obtain an 267 estimate of the avalanche arrival time at each geophone. 268 Avalanche average speed estimates are obtained using the 269 distances involved. Data from GF-6, located on top of the 270 dam (Fig. 1) were of no use because of the spatial 271 distribution of the geophones and the ground character272 istics of the dam (formed by loose gravel with poor 273 vibration transmission).

\section{Results and discussion}

Using the methods described in the previous section (PK and XC) we estimated the speeds for the 11 avalanches at Ryggfonn listed in Table 1. The estimates of the average avalanche propagation speeds using both methods are shown in Fig. 4. To assess the reliability of our methods, we compared our results with the speed measurements from a CW-Doppler radar (Sigurðsson et al., 2004) and with estimates from the arrival time at the load plates (Gauer et al., 2004). In Fig. 4 the average avalanche propagation speeds are represented as a function of the horizontal distance, the steel tower being the origin of the distances. The average avalanche propagation speed between two adjacent geophones is depicted midway between the two sensors to show the evolution of the avalanche along the path. The positions of the sensors are indicated by dots on the path profile in Fig. 4. Averaged speeds obtained from the load plates (steel tower, concrete structure and dam) are plotted midway between the load plates, and speeds from the radar (obtained by averaging the corresponding values from Sigurðsson et al. (2004)) are also indicated in Fig. 4.

In general, there is a reasonable agreement between the different speed estimates. However, the different sensitivity of the different types of equipments used to detect the avalanche, which is reflected in the results, should be pointed out. As regards the LP sensors, the values are obtained from the arrival time of the avalanche at the load cells. This determination is usually easy and unambiguous because of a sudden increase in pressure observed in the record of the LP. Nevertheless, although the strong peaks which are probably produced by the saltation layer or by the dense part of the avalanche are easy to detect, it is much harder to detect the signals generated by the small powder part, which in some cases precedes the dense core. The impacts produced by the saltation layer on the LP are of slightly lower amplitude and higher frequency than that produced by the dense part. The impacts of the powder part are only detected when the LP is not covered by snow. As for the radar, it is also difficult to detect the powder part of small avalanches because of the poor reflectivity of the powder cloud of a low density. Moreover, the estimated speeds obtained by radar are not directly comparable to the front speeds determined by the other methods given that the radar signal is composed of the reflections from many parts of the avalanche body. This is a consequence of the wide angle range from which the radar data are obtained (Gubler, 1987).

As regards the seismic method, previous studies allow us to determine the origin (source) of the seismic signals associated with snow avalanches. It has been confirmed that the powder part is detected despite the low signal amplitude (Suriñach et al., 2001). One of the problems of using seismic sensors to study avalanches is that the origin of the signal, unlike earthquakes is not punctual, but rather it is produced by unlocalized multiple moving sources. As a result the recorded signal from the moving front is contaminated by signals from other parts of the avalanche. These parts also act as sources generating signals that hinder the selection of the arrival times; in our case, the contaminant signals are associated with impacts with the dam and changes in the slope of the path (Suriñach et al., 2001). The application of the RS and an analysis of the seismic time series help us to select (discriminate) the signals associated with the erosion produced by the avalanche front.

When comparing the results obtained by the different methods in Fig. 4 it should be borne in mind that the average speeds are plotted midway between the pair of considered sensors. Overall, the agreement between the results is satisfactory. The speeds estimated using the two seismic approaches (PK and XC) in most of the cases are compatible within the error in the determination of the arrival time over the sensor $(0.4 \mathrm{~s}$ which corresponds to the time resolution of the RS) (Fig. 4). In general, when the wave arrival is clear the PK method is more precise than the XC method. However, for small avalanches and slow wet/dense flows near the dam it was not possible to apply the PK method because of the difficulty of selecting the correct wave arrival by picking. We attribute this difficulty to 1) the presence of the dam, which generates noise in the signal; 2) the low amplitude of the seismic signal detected in the geophones in the dam, probably caused by the large amount of snow covering the geophones which attenuates the signal, and 3) to the low speed or small size of the avalanche, generating little seismic energy. In this case the XC method is more appropriate. Nevertheless, this method may also be unreliable in the presence of very noisy signals. 
As regards the load plates, the speed values are in general consistent with those obtained from the seismic data. The original measurements obtained by radar yield the avalanche speed as a function of time in intervals ranging from 1.5 to $4.5 \mathrm{~s}$. In order to assign a horizontal distance to the radar speed values between two given sensors, these speeds were averaged between the arrival times of the avalanche front over the sensors. These arrival times were extracted from the RS calculations and LP data analysis. The speed values from the radar show differences in relation to the LP and geophones measurements. This was expected given that the geophones and the load plates measure the front speed, whereas the radar records a composite signal proceeding from all the moving parts of the avalanche within the range of the radar. We believed that the averaged speeds correspond to two different parts of the avalanche. Whereas the high speed values from the radar at approx. $100 \mathrm{~m}$ in event 1 (Fig. 4) correspond to the aerosol front of the avalanche (simultaneous pressure measurements show low values (Sigurðsson et al., 2004)), the lower values of the speed obtained from the load plates and seismic sensors correspond to the dense body. In cases where the dense and aerosol parts coexist, geophones detect the former preferentially because of the higher vibrations produced; the same behaviour is observed for the load cells. This is the case for event 2 , which is a dry/mixed avalanche with a large aerosol part and a reduced dense body.

Two different distance evolution profiles of the speed along the path are observed in Fig. 4, which corresponds to the two distinct types of avalanche (dry/mixed and wet). Interestingly, the character of the profile is relatively independent of the size of the avalanche. Dry/mixed avalanches have higher speeds than wet avalanches. If we consider the slope profile in Fig. 4 as a reference curve, all speed values for dry/mixed avalanches are above this curve, whereas the speeds of the wet avalanches are below the curve. Dry/mixed avalanches even seem to accelerate along this part of the path in some cases: The average seismic speed values for the GF2-GF3 interval appear slightly higher than for the GF1-GF2 interval for events 1 and 4, although this is not the case for the radar derived speed values for these events nor for the LP derived speeds for event 1 . Nor is this the case for the wet avalanches where an approximately linear decrease in velocity with distance is observed. The speed values deduced from load plates are also consistent with the observations. Note that the velocity profile of event 7 , which corresponds to the largest dry/mixed avalanche, has a shape similar to that of the other dry/mixed avalanches, but with higher velocities. Similar behaviour is also present in the two different parts of avalanche 8 . The velocity profile corresponding to the first part of the avalanche (8a) displays the behaviour of a dry/mixed avalanche whereas the second part shows a velocity profile of a wet avalanche, as deduced from the analysis of the seismic and LP signals and corroborated by the subsequent field observations. The duration of the wet avalanches in the area of the geophones ranges between 50 and $80 \mathrm{~s}$. The dry/mixed avalanches are shorter and their duration in the area ranges between 8 and $18 \mathrm{~s}$.

\section{Conclusions}

The determination of the speed of an avalanche using the generated seismic signals is possible although a prior detailed seismic analysis is warranted. The complexity of the ground propagation of the energy generated by the avalanche, which may be simulated as a nonpunctual moving source, complicates the seismic time series characteristics. Unlike $\mathrm{N}$ air pressure waves or $\mathrm{P}$ waves, the arrival of the avalanche front does not produce a characteristic, easily identifiable pattern which complicates the picking procedure. The preselection of windows with high amplitudes in the high frequency content of the signal, prior to the determination of the arrival time, proves to be a good tool for seismic phase discrimination. Propagation speed can be obtained from the application of picking (PK) or/and cross-correlation (XC) techniques to the seismic time series. Moreover, these methods are not exclusive, but rather complementary depending on the type of the signal.

The averaged speeds for 11 avalanches at Ryggfonn calculated by applying the PK and XC methods to the seismic time series were compared with speed estimates obtained from load plates and CW-Doppler radar located at the site. In general, a good agreement is found. The discrepancies are mainly related to the different sensitivity of the various instruments. Velocity profiles along the avalanche path were analysed. The two types of avalanches dry/mixed and wet display two different types of profiles regardless of the size of the avalanche. The dry/mixed avalanches propagate faster than the wet avalanches. In two cases, their speed seems to accelerate slightly before sharply decreasing. For the wet avalanches, the velocity decreases approximately linearly with distance. As regards time duration, dry/ mixed type avalanches are as expected shorter. Their duration between the steel tower and the dam ranges between 8 and $18 \mathrm{~s}$, whereas the duration of the wet avalanches in the same area ranges between 50 and $80 \mathrm{~s}$. 

No difference in the behaviour of natural and artificially released avalanches was observed.
Gauer, P., Kristensen, K., Lied, K., 2004. Avalanche studies and model validation in Europe, SATSIE. Ryggfonn measurements Winter 2002/2003 and 2003/2004. Norwegian Geotechnical Institute, Oslo (Norway). 20021048-5.

Granada, F., Marco, O., Villemain, P., 1995. Utilisation de techniques de traitement d'images pour la cartographie des vitesses à la surface d'une avalanche dense. La Houille Blanche 5/6, 66-75.

Gubler, H., 1987. Measurements and modelling of snow avalanche speeds. Avalanche formation, movement and effects. Proceedings of the Davos Symposium, September 1986, vol. 162. IAHS Publ., pp. 405-420.

Harbitz, C., Issler, D., Keylock, C., 1998. Conclusions from a recent survey of avalanche computational models. NGI Publications 203. (Proceedings of 25 Years of Snow Avalanche Research at NGI, Anniversary Conference, Voss, Norway, 12-16 May, 1998). Norwegian Geotechnical Institute, Oslo.

Lied, K., Moe, A., Kristensen, K., Issler, D., 2002. Ryggfonn: Snow Avalanche Research Programme SIP-6: full scale avalanche test site and the effect of the catching dam. NGI Report 581200-35. Norwegian Geotechnical Institute, Oslo, Norway.

McClung, D., Schaerer, P.A., 1993. The Avalanche Handbook, 98. The Mountaineers, 1011 SW Klickitat Way, Seattle, Washington. 134 pp.

McElwaine, J.N., 2004. Calculation of two-dimensional avalanche velocities from opto-electronic sensors. Annals of Glaciology 38, 139-144.

McElwaine, J.N., in press. Rotational flow in gravity current heads, Philosophical Transactions of the Royal Society of London, Series A.

Nishimura, K., Izumi, K., 1997. Seismic signals induced by snow avalanche flow. Natural Hazards 15 (1), 89-100.

Nishimura, K., Maeno, N., Kawada, K., Izumi, K., 1993. Structures of snow cloud in dry-snow avalanches. Annals of Glaciology 18, $173-178$.

Norem, H., Kvisteroy, T.K., Evensen, B.D., 1985. Measurements of avalanche speeds and forces: Instrumentation and preliminary results of the Ryggfonn project. Annals of Glaciology 6, 19-22.

Schaerer, P.A., Salway, A.A., 1980. Seismic and impact-pressure monitoring of flowing avalanches. Journal of Glaciology 26 (94), 179-187.

Sigurðsson, H., Jóhannesson, T., Gubler, H., Kristensen, K., Lied, E., Gauer, P., 2004. Temporary Installation of a CW-Doppler Radar at the Ryggfonn Full-scale Avalanche Test Site in Western Norway. 04025. Icelandic Meteorological Office, Reykjavík (Iceland).

Suriñach, E., Sabot, F., Furdada, G., Vilaplana, J.M., 2000. Study of seismic signals of artificially released snow avalanches for monitoring purposes. Physics and Chemistry of the Earth 25 (9), 721-727.

Suriñach, E., Furdada, G., Sabot, F., Biescas, B., Vilaplana, J.M., 2001. On the characterization of seismic signals generated by snow avalanches for monitoring purposes. Annals of Glaciology 32, 268-274.

Suriñach, E., Vilajosana, I., Khazaradze, G., Biescas, B., Furdada, G., Vilaplana, J.M., 2005. Seismic detection and characterization of landslides and other mass movements. Natural Hazards and Earth System Sciences 5, 1-8 (2005S. Ref-ID: 1684-9981/nhess/2005-5-1). 